TITLE:

\title{
Location-Unbound Color-Shape Binding Representations in Visual Working Memory.
}

$\operatorname{AUTHOR}(\mathrm{S})$ :

Saiki, Jun

\section{CITATION:}

Saiki, Jun. Location-Unbound Color-Shape Binding Representations in Visual Working Memory.. Psychological science 2016, 27(2): 178-190

\section{ISSUE DATE:}

2016-02-09

URL:

http://hdl.handle.net/2433/202916

\section{RIGHT:}

The final, definitive version of this paper has been published in 'Psychological science', February 2016, vol. 27, no. 2 , 178-190, as do:10.1177/0956797615616797 by SAGE Publications Ltd, All rights reserved. (c) The Author(s) 2015.; この論 文は出版社版でありません。引用の際には出版社版をご確認ご利用ください。; This is not the published version. Please cite only the published version. 
Research Article

DOI: $10.1177 / 0956797615616797$

Saiki

Running head: Feature Binding in Visual Working Memory

Human and Environmental Studies, Kyoto University

\section{Corresponding Author:}

Jun Saiki, Kyoto University, Human and Environmental Studies, Yoshida-nihonmatsucho, Sakyo-ku Kyoto 606-8501, Japan

E-mail: saiki@cv.jinkan.kyoto-u.ac.jp

\section{Location-Unbound Color-Shape Binding Representations in Visual Working}

\section{Memory}

Jun Saiki

\section{Abstract}

The mechanism by which nonspatial features, such as color and shape, are bound in visual working memory, and the role of those features' location in their binding, remains unknown. In the current study, I modified a redundancy-gain paradigm to investigate these issues. A set of features was presented in a two-object memory display, followed by a single object probe. Participants judged whether the probe contained any features of the memory display, regardless of its location. Response time distributions revealed feature coactivation only when both features of a single object in the memory display appeared together in the probe, regardless of the response time benefit from the probe and memory objects sharing the same location. This finding suggests that a shared location is 
necessary in the formation of bound representations but unnecessary in their maintenance. Electroencephalography data showed that amplitude modulations reflecting locationunbound feature coactivation were different from those reflecting the location-sharing benefit, consistent with the behavioral finding that feature-location binding is unnecessary in the maintenance of color-shape binding.

\section{Keywords}

visual working memory, feature binding, object file, redundancy gain, event-related potential

Received 11/13/14; Revision accepted 10/19/15

What is the functional unit of visual working memory (VWM) representations? It has been argued that feature-integrated object representations are formed in VWM (Fougnie \& Alvarez, 2011; Luck \& Vogel, 1997; Wheeler \& Treisman, 2002), but if this is the case, how are these feature-bound representations maintained? Do some features play a privileged role? In particular, the role of spatial locations in feature binding has been actively discussed (Kahneman, Treisman, \& Gibbs, 1992; Kondo \& Saiki, 2012; Logie, Brockmole, \& Jaswal, 2011). In the current study, I obtained evidence for shape-color binding representations in VWM unbound to their spatial locations.

Behavioral and neural evidence for integrated VWM representations includes a lack of binding cost in change-detection tasks (Baddeley, Allen, \& Hitch, 2011; Luck \& Vogel, 1997; Saiki \& Miyatsuji, 2007; Wheeler \& Treisman, 2002). In these tasks, objects defined by a single feature and by multiple features show comparable VWM 
capacities. In contrast, some researchers argue against integrated VWM representations, claiming that nonspatial features are maintained independently. For example, during feature-reporting tasks, errors in color and shape reporting are independent (Bays, Wu, \& Husain, 2011; Fougnie \& Alvarez, 2011). In these studies, however, the lack of binding cost and feature-reporting independence were both based on null results.

A direct measurement of feature binding has been developed in perceptualjudgment studies, in which researchers have used the redundancy-gain paradigm to examine feature coactivation. In this paradigm, two target features (e.g., shape and color) are defined, and speeds of perceptual judgment are compared between trials with a single target feature and trials with two target features. Response times (RTs) are faster on twotarget than on single-target trials, a phenomenon called redundancy gain, but this may be accounted for by independent processing of the two features. Miller (1982) proposed the race-model-inequality (RMI) test to evaluate whether the redundancy gain exceeds the limit of independent processing (see the RT analyses section). Violation of the RMI suggests that feature coactivation (i.e., binding of distinct object features in perception) has occurred. During perceptual judgment, coactivation occurs only when two features belong to a single object (Feintuch \& Cohen, 2002; Mordkoff \& Danek, 2011), which indicates that features of a single object are bound together at the perceptual level. If this bound perceptual representation is maintained in VWM, feature coactivation will also be observed in a VWM experiment.

In the present study, the redundancy-gain paradigm was extended to feature binding in VWM. I presented two objects with different colors and shapes in a memory display, and participants were instructed to remember the objects' features. After a 
variable interval, participants judged whether a probe object contained any of the features, regardless of whether the location of the probe matched that of either of the objects in the memory display. The RMI test was conducted with two-feature trials (in which both the color and shape of the probe matched features of the objects in the memory display) and single-feature trials (in which either the color or the shape, but not both, of the probe matched that of the memory objects).

There are conflicting views regarding the role of location in VWM feature binding. Object-file theory (Kahneman et al., 1992) postulates that feature-bound representations are mediated by their spatiotemporal locations (Kondo \& Saiki, 2012; Treisman \& Zhang, 2006). In contrast, some researchers argue that location-unbound representations play significant roles in VWM (Ecker, Mayberry, \& Zimmer, 2013; Logie et al., 2011). This issue was examined in the current study by focusing on the effect of shared location between memory and probe items on feature coactivation. If featurebound VWM is bound to location, feature coactivation would occur only when the probe appeared at the same location as the associated object in the memory display.

To explore the neural correlates of feature coactivation and feature-location binding, I measured brain activity using a scalp electroencephalogram (EEG). Eventrelated potential (ERP) studies comparing memory for previously seen (old) items and unseen (new) items have revealed that old items produce more positive-going waveforms in the N400 component (Schendan \& Kutas, 2007) and that the N400 is sensitive to feature changes between memory and test items (Ecker, Zimmer, \& Groh-Bordin, 2007). Thus, this study focused on the N400 component. The topography of N400 effects revealed distinct patterns associated with location-unbound feature coactivation and with 
location-sharing effects, which suggests that these are mediated by different underlying dynamics.

\section{Experiment 1}

Method

\section{Participants.}

Twenty-six right-handed individuals (mean age $=24$ years, range $=20-34 ; 6$ women, 20 men) with normal color vision and normal or corrected-to-normal visual acuity participated in the experiment. Three of the 26 participants were excluded from analysis because of excessive artifacts in the electrophysiological recordings or high error rates in the behavioral tasks. Sample size was determined using pilot experiments investigating similar effects.

\section{Stimuli.}

Visual stimuli were presented at a distance of $40 \mathrm{~cm}$ on a 17-in. CRT monitor. The stimuli were generated using the Psychophysics Toolbox (Brainard, 1997) designed for MATLAB (The MathWorks, Natick, MA). Each stimulus was one of four novel shapes (Shuman \& Kanwisher, 2004) and was composed of one of four equiluminant colors (red, blue, green, and yellow; $28.0 \mathrm{~cd} / \mathrm{m}^{2}$ ). Stimuli subtended $1.68^{\circ}$. The combination of color and shape was counterbalanced such that all color-shape combinations were presented with equal frequency.

\section{Task and procedure.}

The experiment was conducted in an electrically shielded room. Each trial began with the presentation of two unfilled square boxes, one on either side of a central fixation cross, against a black background (Fig. 1a). These boxes had a width and height of $2.1^{\circ}$, and the 
center of each was $4.0^{\circ}$ away from fixation. After $600 \mathrm{~ms}$, two colored objects were presented, one inside each box, for 200 ms. This was followed by a blank display (boxes alone) that remained visible for a random duration between 400 and 1,200 ms. Next, a single probe object was shown within either the right or the left box until the participant made a response. Participants indicated whether or not the probe object contained any feature (color or shape) presented in the memory display by pressing the right or left button on a response box. The key assignment to “yes” and “no” responses was counterbalanced across participants.

\section{[TS: Please insert Figure 1 about here.]}

The amount of feature matching between probe and memory items varied (Fig. 1b) such that the probe contained two features, one feature, or no features in common with the objects in the memory display. On no-feature trials, the probe did not contain any colors or shapes related to the memory items. The probe for the single-feature trials contained just a single feature of one of the memory items, either its color or its shape. On two-feature trials, the probe contained either both the color and shape of one of the memory items (grouped-features trials) or the color of one object and the shape of the other (separated-features trials).

To investigate the effects of shared location between memory and probe items, I further classified trials in the single-feature condition and grouped-features trials in the two-feature condition into shared-location trials (memory items and probes with matching features appeared in the same box) and unshared-location trials (memory items and probes with matching features appeared in different boxes; Fig. 1b). In all conditions, 
the probe item was in the right hemifield on half of the trials and in the left hemifield on the other half.

Experimental trials were preceded by a 48-trial practice session. The experiment consisted of four separate sessions of 192 trials each. Of the 768 experimental trials, 256 were two-feature, 256 were single-feature, and 256 were no-feature trials.

\section{RT analyses.}

For the RT analyses, trials with incorrect responses and trials on which the RT was shorter than 150 ms or longer than 3,000 ms were excluded. In the redundancy-gain paradigm, RTs are typically faster in the two-feature condition than in the single-feature condition, but this advantage can emerge even when two features are processed independently. The RMI test (Miller, 1982) can help to evaluate whether separateactivation models can account for the redundancy gain using the following inequality: $p(\mathrm{RT}<t \mid$ two-feature trial $) \leq p(\mathrm{RT}<t \mid$ color-match trial $)+p(\mathrm{RT}<t \mid$ shape-match trial $)$, where $t$ equals time.

The RMI expresses the probability that the cumulative density function (CDF) of RTs for the two-feature condition never exceeds the sum of the CDFs of RTs for the color-match and shape-match conditions alone. The RMI defines the upper boundary of RTs in the two-feature condition under all separate-activation models. Therefore, RMI violation (i.e., faster RTs in the two-feature condition than suggested by the RMI) indicates feature coactivation. For quantitative evaluation of RMI, the RT values corresponding to the 5th through 95th percentile (at 5\% intervals) were calculated for the two-feature trials. The RT values for the sum of the percentiles of color-match and shapematch trials that corresponded to the 5th through 95th percentile were also calculated. At 
each quantile, RT difference values (RTs for two-feature trials subtracted from those for the sum of both types of single-feature trials) were examined using a one-tailed $t$ test with the null hypothesis that the difference is zero or a negative value (Mordkoff \& Danek, 2011), and corresponding upper boundaries of 95\% confidence intervals (confidence limits, or CLs) were obtained.

For the analysis of shared-location effects, within each condition, trials with RTs more than \pm 3 standard deviations from each participant’s mean were also excluded. Mean RTs were used for the analysis of shared-location effects

\section{EEG recording.}

EEGs were recorded using a modified international 10-20 system, with $29 \mathrm{Ag} / \mathrm{AgCl}$ electrodes mounted in an elastic cap. All EEG data were recorded in reference to the left earlobe and were rereferenced off-line to the algebraic average of the left and right earlobes. The electrooculogram (EOG) was recorded to detect eye movements and blinks. All electrode impedances were kept below $5 \mathrm{k} \Omega$. The EEG and EOG signals were amplified with a Neurofax $\mu$ EEG-9100 amplifier (Nihon Koden, Tokyo, Japan), bandpass filtered in the range of 0.01 to $120 \mathrm{~Hz}$, and digitized at $500 \mathrm{~Hz}$.

\section{EEG preprocessing.}

The EEG data were processed using custom MATLAB scripts built on the open-source EEGLAB (Delorme \& Makeig, 2004) and FieldTrip (Oostenveld, Fries, Maris, \& Schoffelen, 2011) platforms. Epochs contaminated by blinks and excessive eye movements (i.e., vertical and horizontal EOGs $> \pm 75 \mu \mathrm{V}$ and spike artifacts $> \pm 50 \mu \mathrm{V}$ ) were rejected. On average, $11.1 \%$ of the trials (range $=0 \%-30 \%$ ) were excluded. Epochs associated with incorrect behavioral responses also were excluded from the analyses. 


\section{ERP analyses.}

For statistical analyses of ERP components, I calculated the mean amplitude for each component using a 100-ms baseline period before the probe onset. The N400 component (Kutas \& Federmeier, 2011) was analyzed using the mean amplitude between 300 and $450 \mathrm{~ms}$. The latency of the N400 is known to be quite stable, whereas its topography differs substantially depending on various factors, including stimulus types and tasks (Kutas \& Federmeier, 2011). For example, many studies on semantic processing have revealed that the N400 seems to be in a centro-posterior location, whereas those on recognition memory have found evidence for the N400 in a midfrontal location (where it is often called the FN400).

Therefore, I selected two regions of interest: midfrontal (mean amplitude of the F3, F4, and Fz electrode sites) and midposterior (mean amplitude of the P3, P4, and Pz electrode sites). Since the N400 is a relative component indexing the difference between two conditions (Kutas \& Federmeier, 2011), the main analyses dealt with amplitude differences in both ERP waves and topographies. (Grand-average ERP waveforms are presented in Figs. S1 and S2 in the Supplemental Material available online).

\section{Results}

Table 1 shows the overall mean RT and accuracy in the three main conditions: two feature, single feature (with results for color-match and shape-match trials shown separately), and no feature. RTs were fastest in the two-feature condition, followed by the color-match, shape-match, and no-feature conditions, in that order. The two-feature condition also yielded the highest accuracy, followed by the color-match, no-feature, and 
shape-match conditions, in that order. No trade-off between speed and accuracy was noted. Thus, the following analyses are focused on RTs.

\section{[TS: Please insert Table 1 about here.]}

\section{Feature coactivation of shape and color.}

Figure 2 shows the CDFs of RT data for the two-feature trials and the summed CDFs for the two matching conditions in the single-feature trials. According to the RMI test, the areas where two-feature and summed single-feature curves cross indicates feature coactivation.

\section{[TS: Please insert Figure 2 about here.]}

To examine whether feature coactivation occurs only when target color and shape are grouped together in the probe display, I pooled shared- and unshared-location trials in the single-feature condition and compared them with (a) grouped-features and (b) separated-features trials in the two-feature condition (Fig. 2a). The tests revealed significant feature coactivation only for grouped-features trials, consistent with previous studies using perceptual-judgment tasks. In the grouped-features trials, paired one-tailed $t$ tests revealed a significant violation of RMI at the 5th percentile, $t(22)=4.50, p<.001, d$ $=0.94,95 \% \mathrm{CL}=6.5 ; 10$ th percentile, $t(22)=4.73, p<.001, d=0.99,95 \% \mathrm{CL}=5.2$; and 15th percentile, $t(22)=3.00, p=.003, d=0.63,95 \% \mathrm{CL}=2.2$. No significant violation was observed in the separated-features trials (95\% CLs $=-5.9,-9.4,-11.8$, at the 5th, 10th, and 15th percentiles, respectively).

Object-file theory predicts that within grouped-features trials, feature coactivation should occur only when the probe appears at the same location as the associated memory item. To examine this prediction, I conducted RMI tests separately for shared- and 
unshared-location trials (Fig. 2b). Both shared- and unshared-location trials revealed a significant violation of RMI. In the shared-location trials, the 5th percentile, $t(22)=4.86$, $p<.001, d=1.01,95 \% \mathrm{CL}=10.2$; 10th percentile, $t(22)=5.64, p<.001, d=1.18,95 \%$ CL = 8.9; 15 th percentile, $t(22)=4.55, p<.001, d=0.95,95 \%$ CL $=5.9$; and 20th percentile, $t(22)=2.37, p=.013, d=0.49,95 \% \mathrm{CL}=1.5$, showed significant violation. In the unshared-location trials, the 5th percentile, $t(22)=3.78, p=.001, d=0.79,95 \%$ $\mathrm{CL}=8.0$, and 10th percentile, $t(22)=2.30, p=.016, d=0.48,95 \% \mathrm{CL}=1.6$, showed significant violation. In summary, reliable feature coactivation was observed regardless of location sharing between memory and probe objects, which indicates that sharing a common location across memory and test objects is not a prerequisite for feature coactivation.

\section{Effects of shared location.}

The RMI analysis suggested that contrary to the predictions of object-file theory, feature coactivation does not require binding of color-shape conjunction representation to its location. It might be possible, however, that some property of the current task eliminated the effect of shared location predicted by object-file theory. To test this, I evaluated the effects of shared location by analyzing RTs from color- and shape-match trials in the single-feature condition and grouped-features trials in the two-feature condition (Fig. 3a). A repeated measures analysis of variance (ANOVA) with trial type (two feature, shape match, color match), location sharing (shared, unshared), and probe location (right, left) was conducted. It revealed a significant main effect of trial type, $F(2,44)=74.01, p$ $<.001, \eta_{p}{ }^{2}=.77$, and location sharing, $F(1,22)=13.80, p=.001, \eta_{p}{ }^{2}=.39$, and a significant interaction of trial type and location sharing, $F(2,30)=4.27, p=.020, \eta_{p}{ }^{2}$ 
$=.16$. A simple main effect of location sharing was significant for trials in the twofeature condition, $F(1,22)=11.80, p=.002, \eta_{p}{ }^{2}=.35$, and for shape-match trials, $F(1$, 22) $=12.63, p=.002, \eta_{p}{ }^{2}=.36$, but was not significant for color-match trials, $F(1,22)=$ $0.46, p>.250, \eta_{p}^{2}=.02$.

\section{[TS: Please insert Figure 3 about here.]}

Overall, behavioral RTs showed reliable effects of shared location in the twofeature and shape-match conditions, but the effect in the color-match condition was small and unreliable. The shared location facilitated RTs, but this effect was not a prerequisite for the feature coactivation of color and shape in the current experiment.

\section{ERP analysis.}

The analyses of ERPs triggered at the onset of the probe stimulus explored the neural correlates of feature coactivation and shared-location effects. Following the N400 studies on recognition memory, which showed positive modulation for old relative to new items, I defined the ERP difference waveforms by subtracting the separated-features from the grouped-features trials for the effect of feature coactivation; for the location-sharing effect, I subtracted the unshared-location from the shared-location trials. Thus, significantly positive difference waveforms indicate significant effects of the N400 component. Figure 4 shows the scalp topography and waveforms of N400 amplitude differences, separately for the effect of feature coactivation and for the effect of location sharing. For feature coactivation, there was a significantly larger amplitude in the midfrontal than in the midposterior region, $t(22)=2.70, p=.013, d=0.56,95 \%$ confidence interval $(\mathrm{CI})=[0.16,1.24]$. The midfrontal region showed a significantly 
positive amplitude, $t(22)=2.62, p=.016, d=0.55,95 \% \mathrm{CI}=[0.15,1.26]$, whereas the midposterior region did not, $t(22)=0.02, p>.250, d=0.003, \mathrm{CI}=[-0.56,0.57]$.

\section{[TS: Please insert Figure 4 about here.]}

Regarding the effect of location sharing, there was a significantly larger amplitude in the midposterior than in the midfrontal region, $t(22)=2.50, p=.020, d=0.52,95 \% \mathrm{CI}$ $=[0.18,1.91]$. The midposterior region showed a significantly positive amplitude, $t(22)=$ 4.00, $p<.001, d=0.83,95 \% \mathrm{CI}=[0.66,2.08]$, whereas the midfrontal region did not, $t(22)=0.86, p>.250, d=0.18, \mathrm{CI}=[-0.46,1.10]$.

\section{Discussion.}

Taken together, the results of Experiment 1 revealed feature coactivation only in the grouped-features condition, regardless of location sharing between memory and probe items. The results of ERP analysis were consistent with the behavioral data. However, the redundancy gain obtained in Experiment 1 may be contaminated by interference in the single-feature trials because of the use of target features as nontargets. In the perceptual redundancy-gain paradigm, nontarget features never become targets, because target and nontarget features are predetermined and fixed throughout the experiment. In Experiment 1 , in contrast, a nontarget feature in a single-feature trial could become a target feature in another trial, because target features were determined on a trial-by-trial basis. Thus, the redundancy gain in Experiment 1 may involve RT delay in the single-feature trials because of suppression of the nontarget feature rather than activation of the target feature.

\section{Experiment 2}

To eliminate the possible interference effect in Experiment 1, I created a pair of nontarget features for Experiment 2 that were never presented in the memory display. 


\section{Method}

The method in Experiment 2 was identical to that of Experiment 1, except that a nontarget color and a nontarget shape were added to the stimuli, and EEG was not recorded.

\section{Participants.}

Twenty-four right-handed individuals (mean age $=22$ years, range $=18-32 ; 6$ women, 18 men) participated in the experiment. Data were not obtained from 1 participant because of equipment malfunction.

\section{Stimuli and procedure.}

Visual stimuli were presented at a distance of $57 \mathrm{~cm}$ on a 21-in. CRT monitor; thus, the size of each object was $1.18^{\circ}$. To eliminate the effect of interference in the single-feature trials, I added a nontarget color (gray) and nontarget shape (blurred circle), which appeared only in the probe display. The four target colors and shapes were the same as those used in Experiment 1.

Two- and single-feature trials were the same as in Experiment 1, except that the probe object in the single-feature trials always combined a target feature and a nontarget feature. There were two types of no-feature trials: combinations of target color and shape not presented in the memory display, as in Experiment 1, and the combination of nontarget color and shape, namely, a gray blurred circle. The no-feature trials consisting of target features were necessary because otherwise participants could respond "yes” to any probe objects other than the gray blurred circle. If the redundancy gain in Experiment 1 was due to interference by the distractor feature, the RMI test in Experiment 2 should 
not show the violation. If the redundancy gain reflects feature coactivation, the RMI test in Experiment 2 should show the violation.

Experimental trials were preceded by a 48-trial practice session. There were 16 blocks of 48 trials (768 trials total): 256 two feature, 256 single feature, and 256 no feature.

\section{Results}

Two of the 23 participants were excluded from analyses because of high false alarm rates (more than $15 \%$ of all trials); they were suspected of responding "yes” to any features other than gray and the blurred circle. The RMI test (Fig. 5a) revealed significant feature coactivation only on grouped-features trials, consistent with Experiment 1. In these trials, paired one-tailed $t$ tests revealed a significant violation of RMI at the 5th percentile, $t(20)$ $=1.77, p=.046, d=0.39,95 \% \mathrm{CL}=0.1$, and 15th percentile, $t(20)=2.14, p=.023, d=$ $0.47,95 \% \mathrm{CL}=1.1$. No significant violation was observed in the separated-features trials (95\% CLs $=-13.1,-12.6,-9.4$, at the 5th, 10th, and 15th percentiles, respectively).

\section{[TS: Please insert Figure 5 about here.]}

Similar to Experiment 1, both shared- and unshared-location trials revealed a significant violation of RMI (Fig. 5b). In the shared-location trials, the 5th percentile, $t(20)=2.26, p=.018, d=0.49,95 \% \mathrm{CL}=3.3$; 10th percentile, $t(20)=1.96, p=.032, d=$ 0.43, 95\% CL $=1.0$; and 15th percentile, $t(20)=2.09, p=.025, d=0.46,95 \%$ CL $=1.3$, showed significant violation. In the unshared-location trials, the 5th percentile, $t(22)=$ 2.73, $p=.007, d=0.59,95 \% \mathrm{CL}=5.7$, showed significant violation. The same pattern of feature coactivation was observed without interference in the single-feature trials, which 
indicates that the results of Experiment 1 are not entirely due to interference from distractor features.

The effects of shared location were similar to those in Experiment 1 (see Fig. 2b). A repeated measures ANOVA revealed a significant main effect of trial type, $F(2,40)=$ 29.33, $p<.001, \eta_{p}{ }^{2}=.59$, and location sharing, $F(1,20)=19.45, p<.001, \eta_{p}{ }^{2}=.49$, and a marginally significant interaction of trial type and location sharing, $F(2,40)=2.80, p$ $=.073, \eta_{p}^{2}=.12$. A simple main effect of location sharing was significant in the twofeature condition, $F(1,20)=4.98, p=.037, \eta_{p}{ }^{2}=.20$, and shape-match condition, $F(1$, 20) $=33.24, p<.001, \eta_{p}{ }^{2}=.62$, but was marginally significant in the color-match condition, $F(1,20)=4.11, p=.056, \eta_{p}^{2}=.17$.

\section{General Discussion}

The current study provides evidence for color-shape conjunction memory in VWM using a modified redundancy-gain paradigm. The RMI test revealed that feature coactivation occurs only when color and shape belong to a single object. More important, it occurs regardless of location sharing between memory and probe objects, which suggests that color-shape conjunction representations are not bound to their locations. Experiment 2 eliminated the possibility that interference solely accounts for the feature-coactivation effect. The effect of location sharing was observed, but was somewhat weaker, on colormatch trials than on shape-match and two-feature trials. ERP analysis revealed that amplitude modulations for feature coactivation of color and shape and for the effect of shared location have different topographic distributions. Differential topographies in ERPs are consistent with behavioral results showing that feature-location binding is not a necessary condition for color-shape binding. 


\section{Feature binding in VWM}

The current study provides unequivocal evidence for the binding of color and shape in VWM. Previous studies supporting binding in VWM have revealed the lack of binding costs (Luck \& Vogel, 1997; Luria \& Vogel, 2011) and dual-task costs (Baddeley et al., 2011). However, the lack of task costs can be accounted for by a disjunction of featurebased VWM (Wheeler \& Treisman, 2002). As far as binding across different visual attributes such as color and shape, previous data cannot refute independent operation of color-based and shape-based VWM systems (Bays et al., 2011; Fougnie \& Alvarez, 2011). In contrast, feature coactivation revealed by the RMI test eliminates the possibility of probability summation of independent feature-based VWM.

The discrepancy between the results found in the current study and in some recent studies reporting a lack of binding (Bays et al., 2011; Fougnie \& Alvarez, 2011) may be accounted for by task differences. Fougnie and Alvarez (2011) showed independence of feature-reporting errors in the continuous-report task (Zhang \& Luck, 2008) using objects with color and orientation. They argue that binding in VWM should lead to correlation in errors of color and orientation, and the lack of correlation supports the view of a featurebased VWM system. These studies used a task in which the location of objects was relevant, thus VWM representations bound to location were investigated. The current study failed to show a neural correlate of feature coactivation in the region showing a location-binding effect (i.e., midposterior), which suggests that color-location and shapelocation binding memories are independently maintained. This result is consistent with the lack of color-shape interaction in location-bound memory retrieval in previous studies. Location-irrelevant tasks may be sensitive to color-shape binding representations, 
whereas location-relevant tasks may primarily use single-feature VWM bound to locations.

Recently, a study comparing intrinsic and extrinsic binding in VWM revealed a filtering cost of task-irrelevant color in a shape-memory task only under intrinsic binding conditions (Ecker et al., 2013). Task performance was better when the probe shape was presented with the same color as in the memory display and worse when the color was recombined. The current study provides complementary evidence using redundancy gain as a measure, indicating that maintenance of intrinsic color-shape binding representations is general across different tasks. By manipulating location sharing between memory and probe objects, I further showed that color-shape binding representations are unbound to location.

\section{Type and token representations in VWM}

Object-file theory (Kahneman et al., 1992) postulates that episodic representations of visual objects play critical roles in visual cognition and that spatiotemporal location is necessary to form and maintain object files. Object representations bound and unbound to locations are often called tokens and types, respectively. Empirical studies on object files (Kahneman et al., 1992; Noles, Scholl, \& Mitroff, 2005) have never directly investigated multifeature objects; thus, the nature of multifeature type and token representations remains unknown. The current study showed that feature coactivation occurs regardless of location sharing, which indicates that activation of feature-bound VWM representation does not require addressing an object's location.

Note that the current study does not directly address the role of location in forming multifeature object representations. Indeed, the significant advantage of the two- 
feature grouped condition over the two-feature separated condition shows the importance of spatial location in memory formation. This grouping advantage may reflect an explicit binding to locations in memory formation by attentive processing as postulated in feature-integration theory (Treisman \& Gelade, 1980) or an implicit role of locations in forming a type representation of multiple features. Regardless of the role of location in memory formation, the current study provides evidence that a multifeature-type representation becomes available during VWM maintenance.

The current study failed to provide evidence for color-shape feature coactivation specific to token VWM. Note that the shared-location effects observed in the two-feature condition provide insufficient evidence for object files for multifeature objects, because they may simply reflect independent effects of color-location and shape-location bindings. This finding raises an important issue regarding the nature of the object-file system. This system may be able to bind only simple object representations, possibly single features, to spatiotemporal locations. Such a constraint is consistent with a severe limitation in object-tracking ability (Saiki, 2003) and the lack of correlation in feature-report tasks (Bays et al., 2011; Fougnie \& Alvarez, 2011). Hollingworth and Rasmussen (2010) reported a similar limitation in the object-file system and proposed a scene-based VWM as a complementary mechanism. Whether scene-based VWM can account for these findings remains unknown, and further investigations, including object-motion manipulation, are necessary.

\section{ERP components related to feature binding}

ERP analysis of trials in the two-feature condition revealed significantly positive N400 amplitude differences in the midfrontal region for color-shape feature coactivation and in 
the midposterior region for the shared-location effect. With a wide variety of stimuli and tasks, the N400 component shows more positive modulation for items congruent with than those incongruent with memory representations (Kutas \& Federmeier, 2011). For example, old items in recognition memory (Curran, Tanaka, \& Weiskopf, 2002), conceptual match in priming (Voss \& Paller, 2006), and perceptual match in object categorization (Schendan \& Kutas, 2007) all show positive modulation in the N400. The current study also revealed that the higher degree of match between perceptual and memory representations leads to more positive ERP amplitudes.

Two important novel findings deserve discussion. First, a neural correlate of the shared-location effect in the N400, not in later components, suggests that effects of shared location occur temporally parallel with location-unbound feature coactivation. Although many theoretical models assume that activation of type representation precedes binding to location, called tokenization (Kanwisher, 1987), the current results suggest that type and token representations may be processed in parallel. Second, N400 modulations by the location-unbound color-shape coactivation and location binding show quite different scalp topographies. The topography difference may indicate different cognitive mechanisms, but careful consideration is necessary. The VWM task in the current study differs from tasks relying on long-term memory typical in the N400 literature. It contains aspects of recognition memory and priming, which may induce effects related to both tasks. These methodological differences may obscure clear interpretations of ERP topography. However, the current study shows, for the first time, that the N400 component is a useful index in understanding feature binding in VWM. Further studies are needed to elucidate underlying neural mechanisms. 


\section{Conclusion}

The novel approach in the current study of combining redundancy-gain and objectreviewing paradigms can provide unequivocal evidence for shape-color binding in VWM. Maintenance of shape-color binding in VWM unbound to location is mediated by activity in the frontal region, distinct from maintenance of feature-location bindings mediated by activity in the posterior region. Color-shape binding is maintained without binding to location, which provides no clear evidence for binding of color-shape conjunction to location. These novel findings pose constraints on the existing theoretical models of VWM and suggest a parallel maintenance of type and token VWM representations.

\section{Author Contributions}

J. Saiki is the sole author of this article and is responsible for its content.

\section{Acknowledgments}

I thank Keisuke Fukuda and Daryl Fougnie for comments on an earlier manuscript and Hiroki Koga, Qi Li, and Chisato Mine for data collection.

\section{Declaration of Conflicting Interests}

The author declared that he had no conflicts of interest with respect to his authorship or the publication of this article.

\section{Funding}

This work was supported by Grant Nos. 21300103 and 24240041 from the Grants-in-Aid for Scientific Research (KAKENHI) program of the Japan Society for the Promotion of Science and by a Global Centers of Excellence Program grant (D07 to Kyoto University) from Japan’s Ministry of Education, Culture, Sports, Science and Technology. 


\section{Supplemental Material}

Additional supporting information can be found at

http://pss.sagepub.com/content/by/supplemental-data

\section{References}

Baddeley, A. D., Allen, R. J., \& Hitch, G. J. (2011). Binding in visual working memory: The role of the episodic buffer. Neuropsychologia, 49, 1393-1400.

Bays, P. M., Wu, E. Y., \& Husain, M. (2011). Storage and binding of object features in visual working memory. Neuropsychologia, 49, 1622-1631.

Brainard, D. H. (1997). The Psychophysics Toolbox. Spatial Vision, 10, 433-436.

Curran, T., Tanaka, J. W., \& Weiskopf, D. M. (2002). An electrophysiological comparison of visual categorization and recognition memory. Cognitive, Affective, \& Behavioral Neuroscience, 2, 1-18.

Delorme, A., \& Makeig, S. (2004). EEGLAB: An open source toolbox for analysis of single-trial EEG dynamics. Journal of Neuroscience Methods, 134, 9-21.

Ecker, U. K. H., Mayberry, M., \& Zimmer, H. D. (2013). Binding of intrinsic and extrinsic features in working memory. Journal of Experimental Psychology: General, 142, 218-234.

Ecker, U. K. H., Zimmer, H. D., \& Groh-Bordin, C. (2007). Color and context: An ERP study on intrinsic and extrinsic feature binding in episodic memory. Memory \& Cognition, 35, 1483-1501.

Feintuch, U., \& Cohen, A. (2002). Visual attention and coactivation of response decisions for features from different dimensions. Psychological Science, 13, 361-369. 
Fougnie, D., \& Alvarez, G. A. (2011). Object features fail independently in visual working memory: Evidence for a probabilistic feature-store model. Journal of Vision, 11(12), Article 3. doi:10.1167/11.12.3

Hollingworth, A., \& Rasmussen, I. P. (2010). Binding objects to locations: The relationship between object files and visual working memory. Journal of Experimental Psychology: Human Perception and Performance, 36, 543-564.

Kahneman, D., Treisman, A., \& Gibbs, B. J. (1992). The reviewing of object files: Object-specific integration of information. Cognitive Psychology, 24, 175-219.

Kanwisher, N. G. (1987). Repetition blindness: Type recognition without token individuation. Cognition, 27, 117-143.

Kondo, A., \& Saiki, J. (2012). Feature-specific encoding flexibility in visual working memory. PLoS ONE, 7(12), Article e50962. doi:10.1371/journal.pone.0050962

Kutas, M., \& Federmeier, K. D. (2011). Thirty years and counting: Finding meaning in the N400 component of the event-related brain potential (ERP). Annual Review of Psychology, 61, 621-647.

Logie, R. H., Brockmole, J. R., \& Jaswal, S. (2011). Feature binding in visual short-term memory is unaffected by task-irrelevant changes of location, shape, and color. Memory \& Cognition, 39, 24-36.

Luck, S. J., \& Vogel, E. K. (1997). The capacity of visual working memory for features and conjunctions. Nature, 390, 279-281.

Luria, R., \& Vogel, E. K. (2011). Shape and color conjunction stimuli are represented as bound objects in visual working memory. Neuropsychologia, 49, 1632-1639. 
Miller, J. (1982). Divided attention: Evidence for coactivation with redundant signals. Cognitive Psychology, 14, 247-279.

Mordkoff, J. T., \& Danek, R. H. (2011). Dividing attention between color and shape revisited: Redundant targets coactivate only when parts of the same perceptual object. Attention, Perception, \& Psychophysics, 73, 103-112.

Noles, N. S., Scholl, B. J., \& Mitroff, S. R. (2005). The persistence of object file representations. Perception \& Psychophysics, 67, 324-334.

Oostenveld, R., Fries, P., Maris, E., \& Schoffelen, J. M. (2011). FieldTrip: Open source software for advanced analysis of MEG, EEG, and invasive electrophysiological data. Computational Intelligence and Neuroscience, 2011, Article 156869. doi:10.1155/2011/156869

Saiki, J. (2003). Spatiotemporal characteristics of dynamic feature binding in visual working memory. Vision Research, 43, 2107-2123.

Saiki, J., \& Miyatsuji, H. (2007). Feature binding in visual working memory evaluated by type identification paradigm. Cognition, 102, 49-83.

Schendan, H. E., \& Kutas, M. (2007). Neurophysiological evidence for the time course of activation of global shape, part, and local contour representations during visual object categorization and memory. Journal of Cognitive Neuroscience, 19, 734749.

Shuman, M., \& Kanwisher, N. (2004). Numerical magnitude in the human parietal lobe: Tests of representational generality and domain specificity. Neuron, 44, 557-569. Treisman, A., \& Gelade, G. (1980). A feature-integration theory of attention. Cognitive Psychology, 12, 97-136. 
Treisman, A., \& Zhang, W. (2006). Location and binding in visual working memory. Memory \& Cognition, 34, 1704-1719.

Voss, J. L., \& Paller, K. A. (2006). Fluent conceptual processing and explicit memory for faces are electrophysiologically distinct. Journal of Neuroscience, 26, 926-933.

Wheeler, M. E., \& Treisman, A. M. (2002). Binding in short-term visual memory. Journal of Experimental Psychology: General, 131, 48-64.

Zhang, W., \& Luck, S. J. (2008). Discrete fixed-resolution representations in visual working memory. Nature, 453, 233-235. 
Table 1.

Mean Response Time (RT) and Accuracy in Experiments 1 and 2

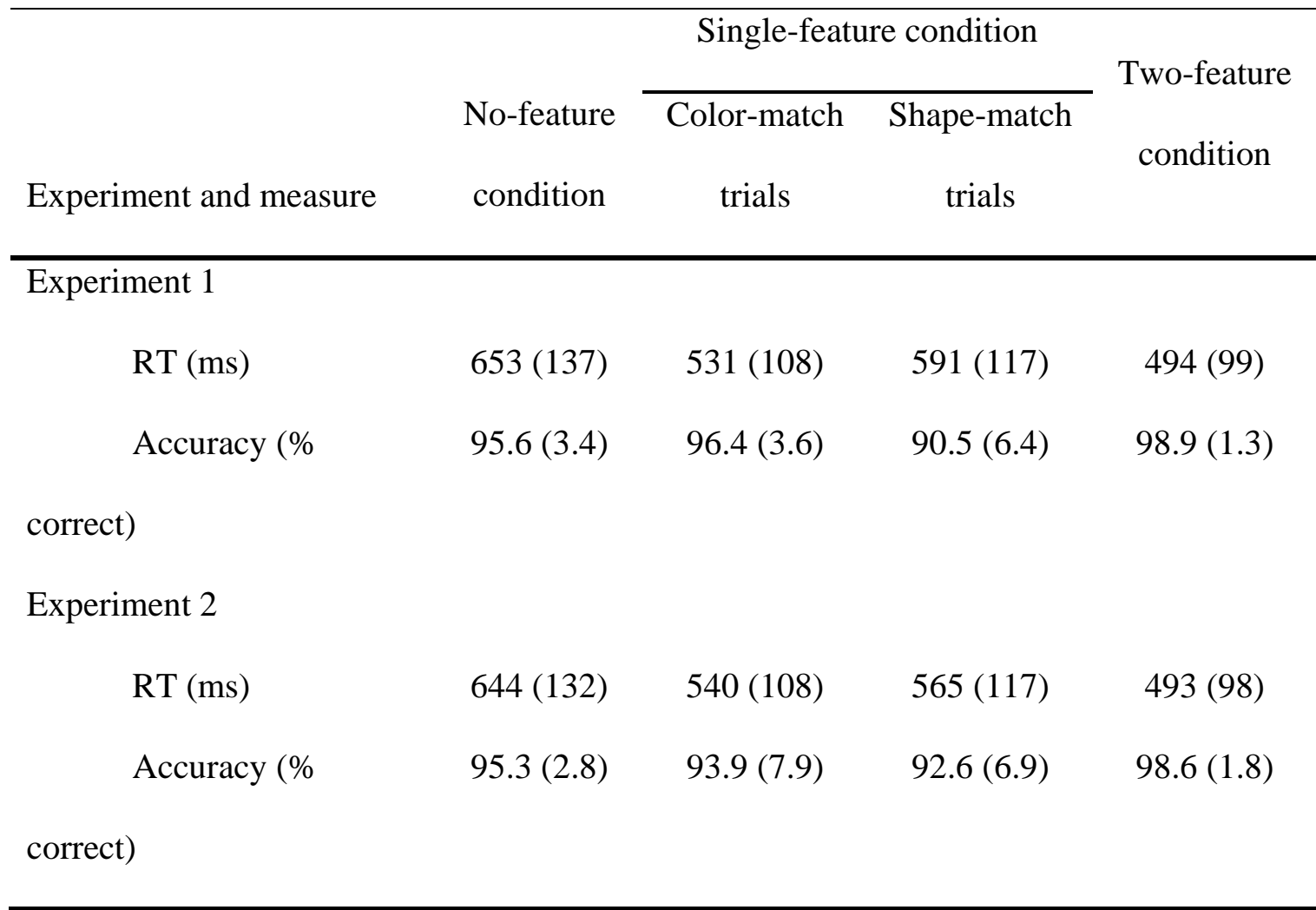

Note: Standard deviations are given in parentheses.

Figure legends

Fig. 1.

Example trial sequence and design from Experiment 1. Trials started with a blank display containing two boxes as placeholders (a), followed by a memory display in which objects with different features (color and shape) appeared in each box. When the probe display appeared, participants had to indicate whether the single object in the display contained any of the features of the objects in the memory display. The probe could have two 
features, one feature, or no features in common with the memory items (b). In the twofeature condition, probes could share both the color and shape of one of the memory items (grouped-features trials) or the color of one memory item and the shape of the other (separated-features trials). In the single-feature condition, either the color or the shape matched that of one of the memory items. In the no-feature condition, probe and memory items had no features in common. Trials in the two- and single-feature conditions were further separated into those on which the location of the probe and the associated feature or features of the memory item was shared and those on which the location was unshared.

\section{Fig. 2.}

Cumulative distribution functions (CDFs) from Experiment 1. Each graph shows the probability for response time (RT), separately for the two-feature condition and for the sum of the CDFs in the color- and shape-match trials in the single-feature condition. Areas where curves cross indicate feature coactivation. The top row (a) shows results when features of the probe and memory items in the two-feature condition were grouped or separated, and the bottom row (b) shows results for shared- and unshared-location trials. Asterisks indicate a significant difference between CDFs for each condition $\left({ }^{*} p\right.$ $<.05,{ }^{* *} p<.01$, as determined with paired-samples $t$ tests).

\section{Fig. 3.}

Mean response time as a function of trial type and location-sharing condition, separately for (a) Experiment 1 and (b) Experiment 2. Symbols indicate significant differences 
between conditions $\left({ }^{\dagger} p<.10,{ }^{*} p<.05\right.$, $\left.{ }^{* *} p<.01\right)$. Error bars indicate $95 \%$ confidence limits.

Fig. 4.

Event-related potential (ERP) results from the two-feature condition in Experiment 1: scalp topography of N400 difference signals (a, c) and grand-average ERP difference waveforms for the midfrontal and midposterior electrode sites (b, d). In the top row, results are shown for the N400 difference between grouped- and separated-features trials (grouped - separated); in the bottom row, results are shown for the N400 difference between shared- and unshared-location trials (shared - unshared). Shaded areas denote periods during which mean N400 difference amplitudes were calculated (300-450 ms after probe onset). Asterisks indicate a significant difference between the ERP differences at the two electrode sites $\left({ }^{*} p<.05\right.$, as determined with paired-samples $t$ tests).

Fig. 5.

Cumulative distribution functions (CDFs) from Experiment 2. Each graph shows the probability for response time (RT), separately for the two-feature condition and for the sum of the CDFs in the color- and shape-match trials in the single-feature condition. Areas where curves cross indicate feature coactivation. The top row (a) shows results when features of the probe and memory items in the two-feature condition were grouped or separated, and the bottom row (b) shows results for shared and unshared-location trials. Asterisks indicate a significant difference between CDFs for each condition $\left({ }^{*} p<.05\right.$, ${ }^{* *} p<.01$, as determined with paired-samples $t$ tests). 
a

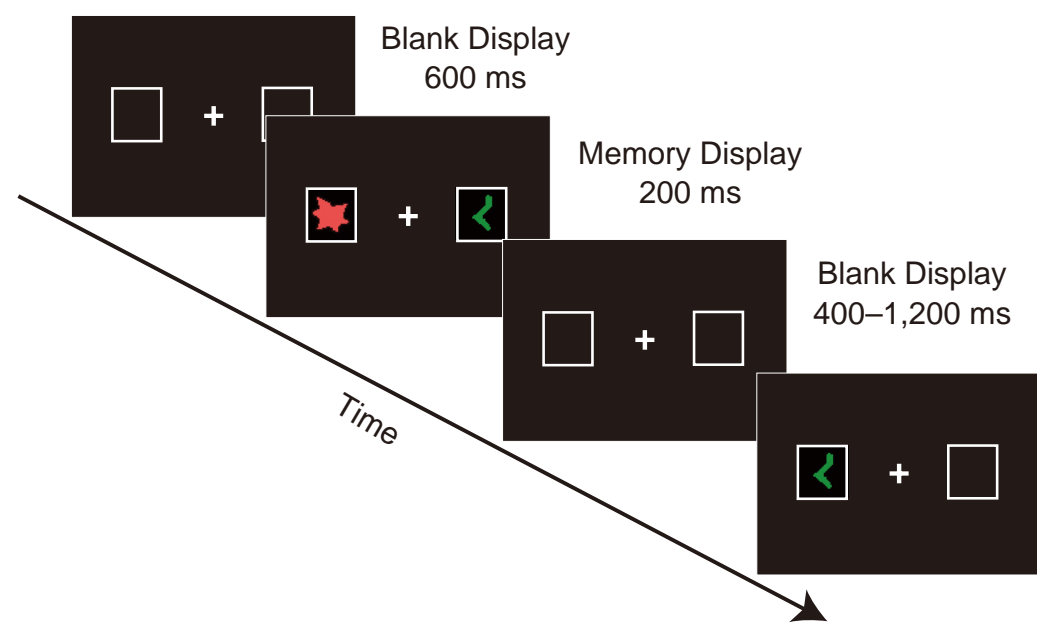

Probe Display

Until Response

b

Two-Feature Condition

Grouped Features

Shared Unshared Mixed

Not

Location

Location

Location

Applicable

Single-Feature Condition

Color Match

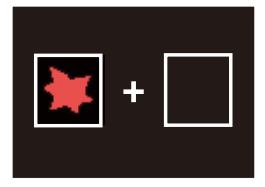

Separated Features

Single-Feature Condition

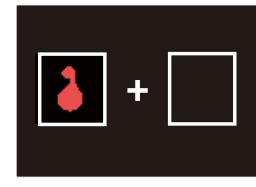

Shape Match
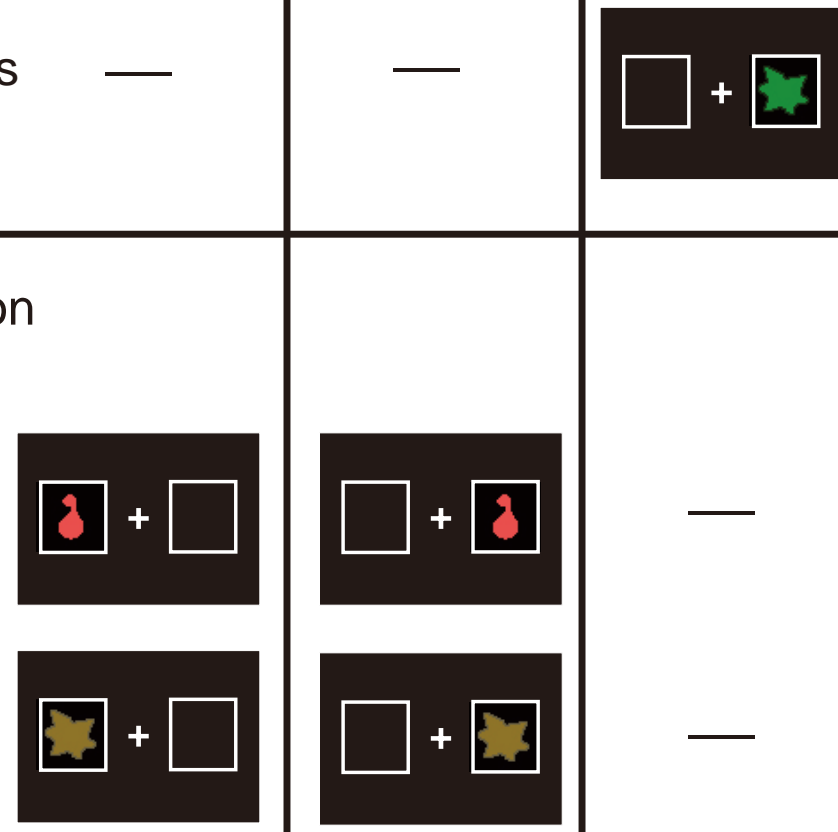

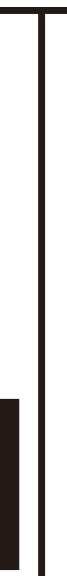

Shape Match
No-Feature Condition


a

\section{Grouped Condition}

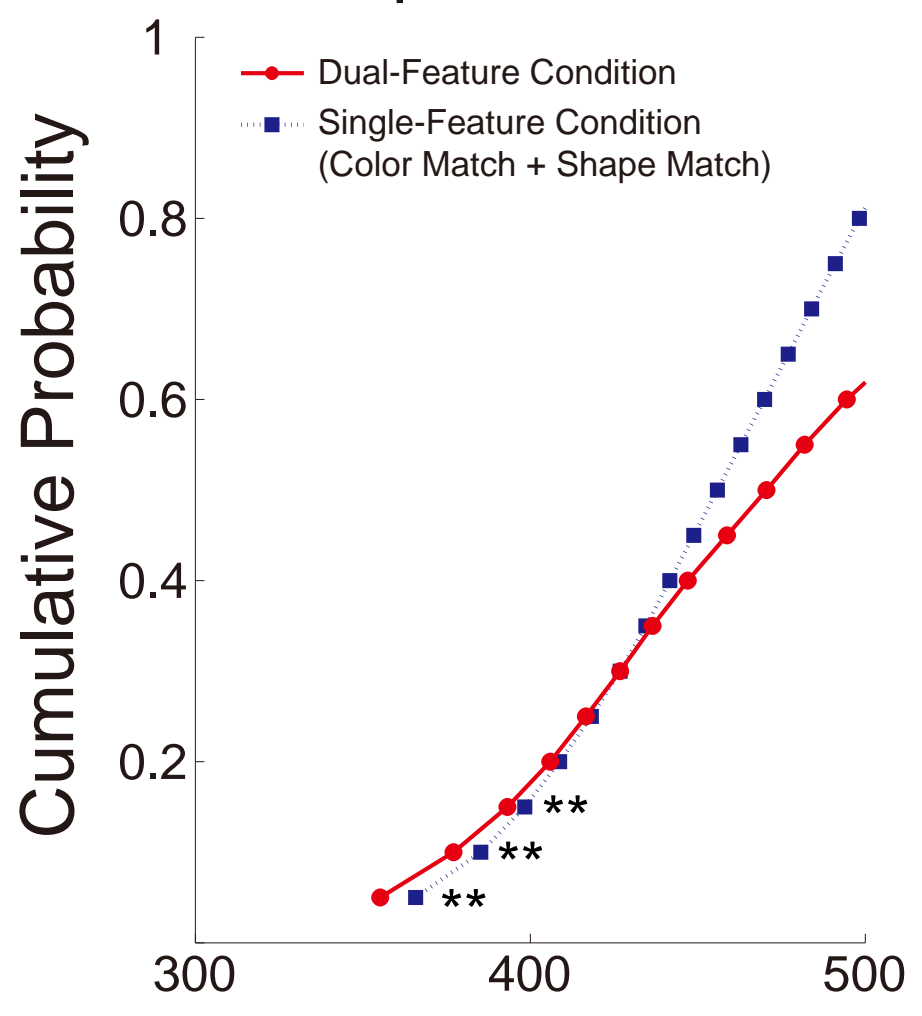

Response Time (ms)
Separated Condition

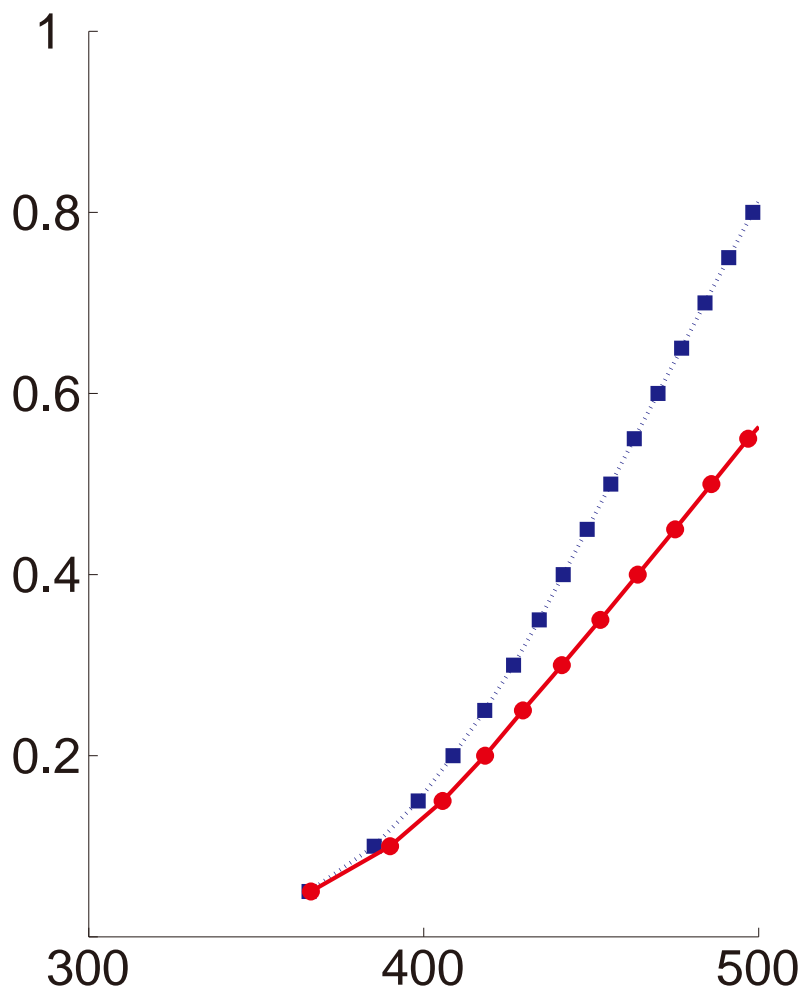

Response Time (ms)

b

Location-Shared

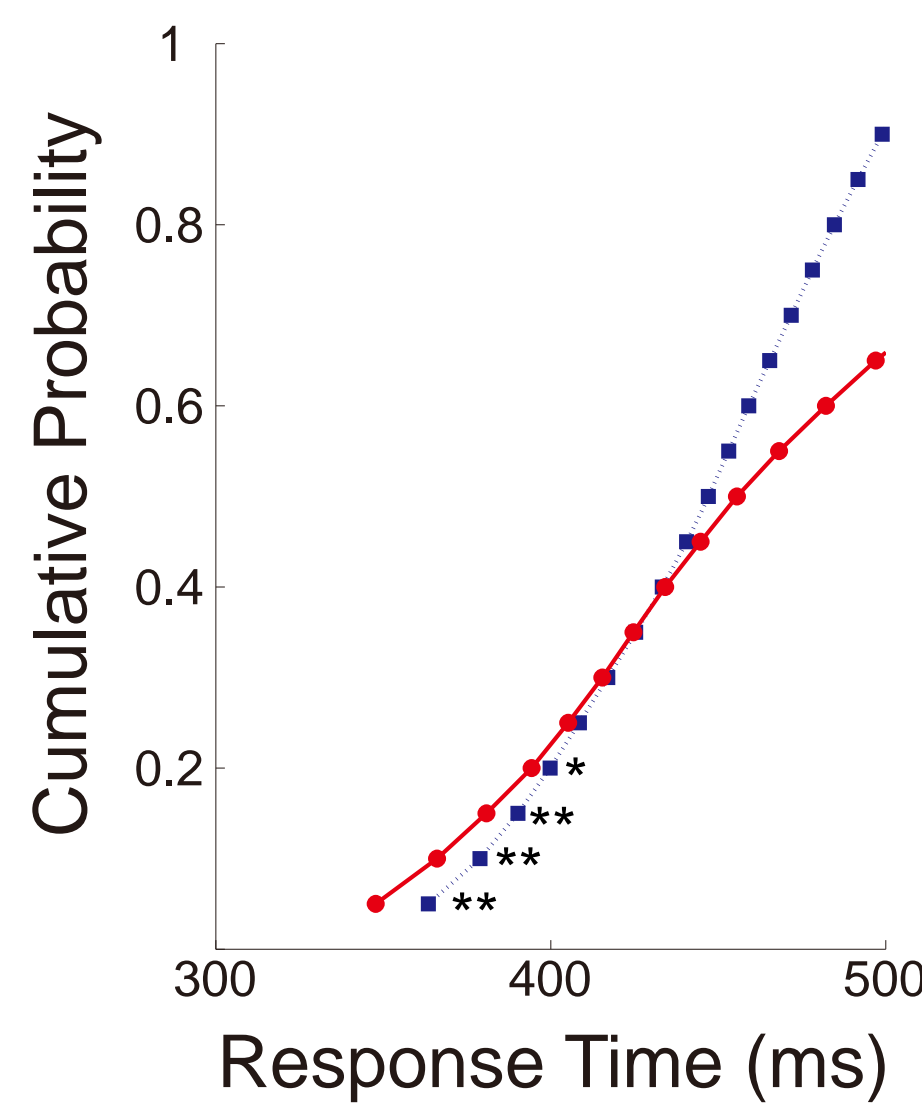

Location-Unshared

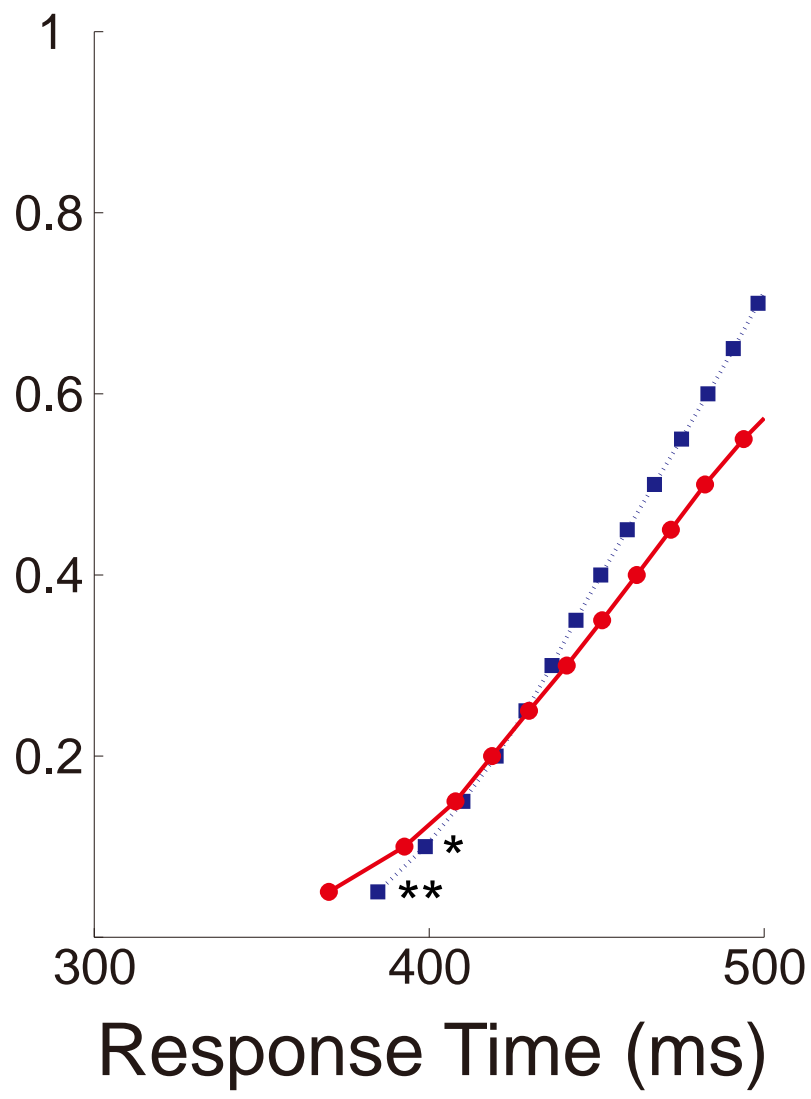




\section{a $\square$ Unshared-Location Condition b}
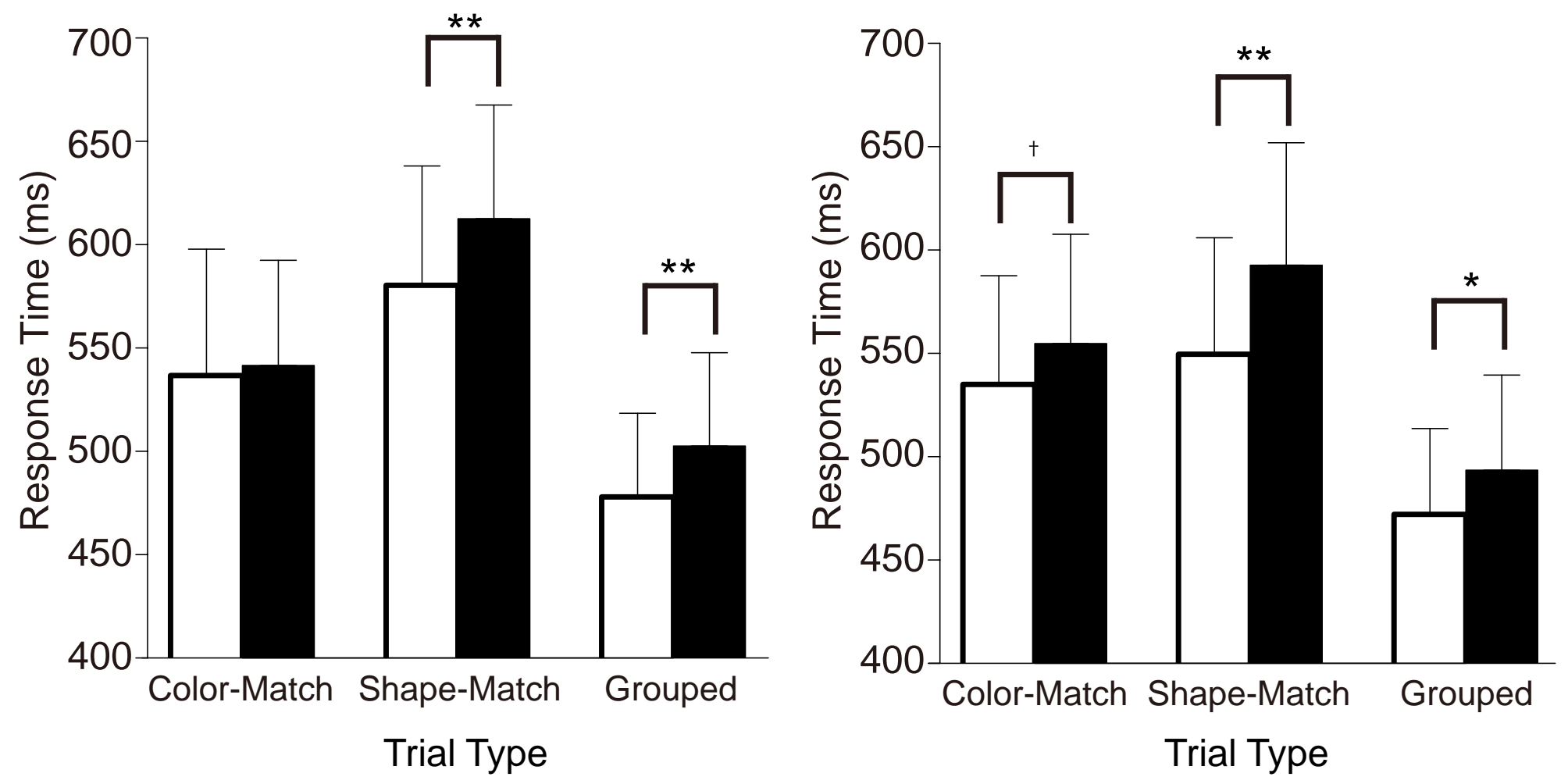
a

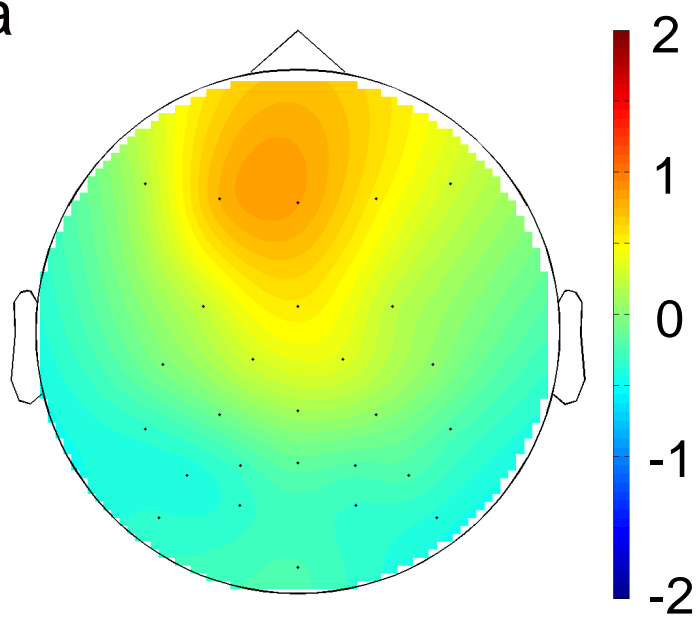

C

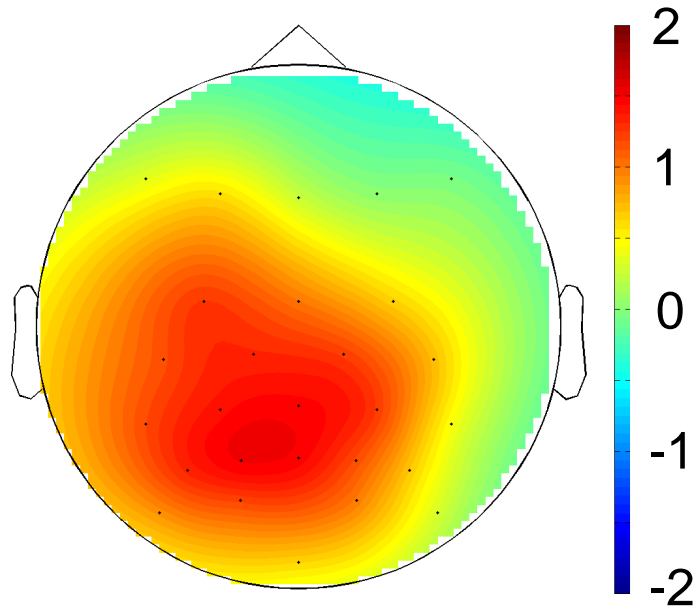

b

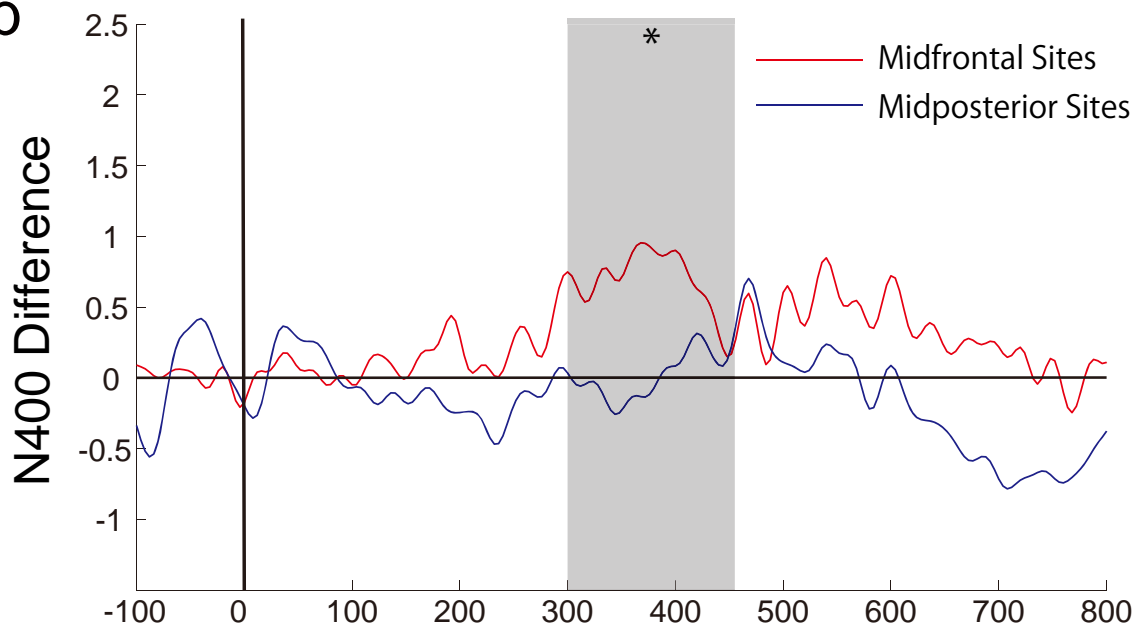

d

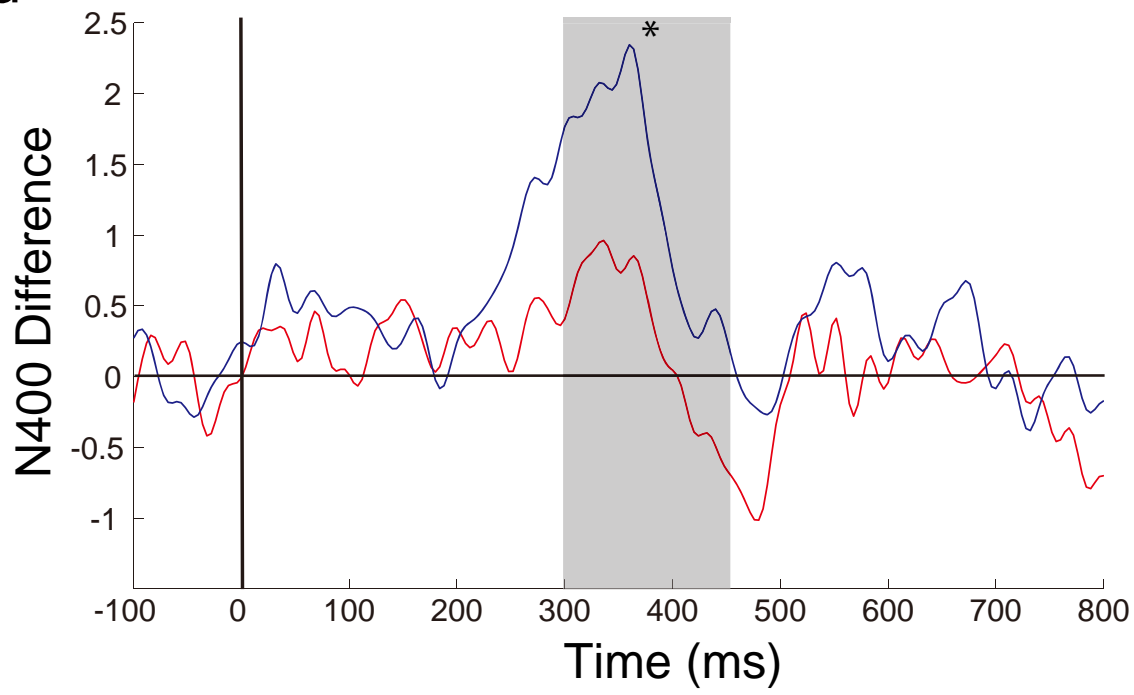


A.

Grouped condition

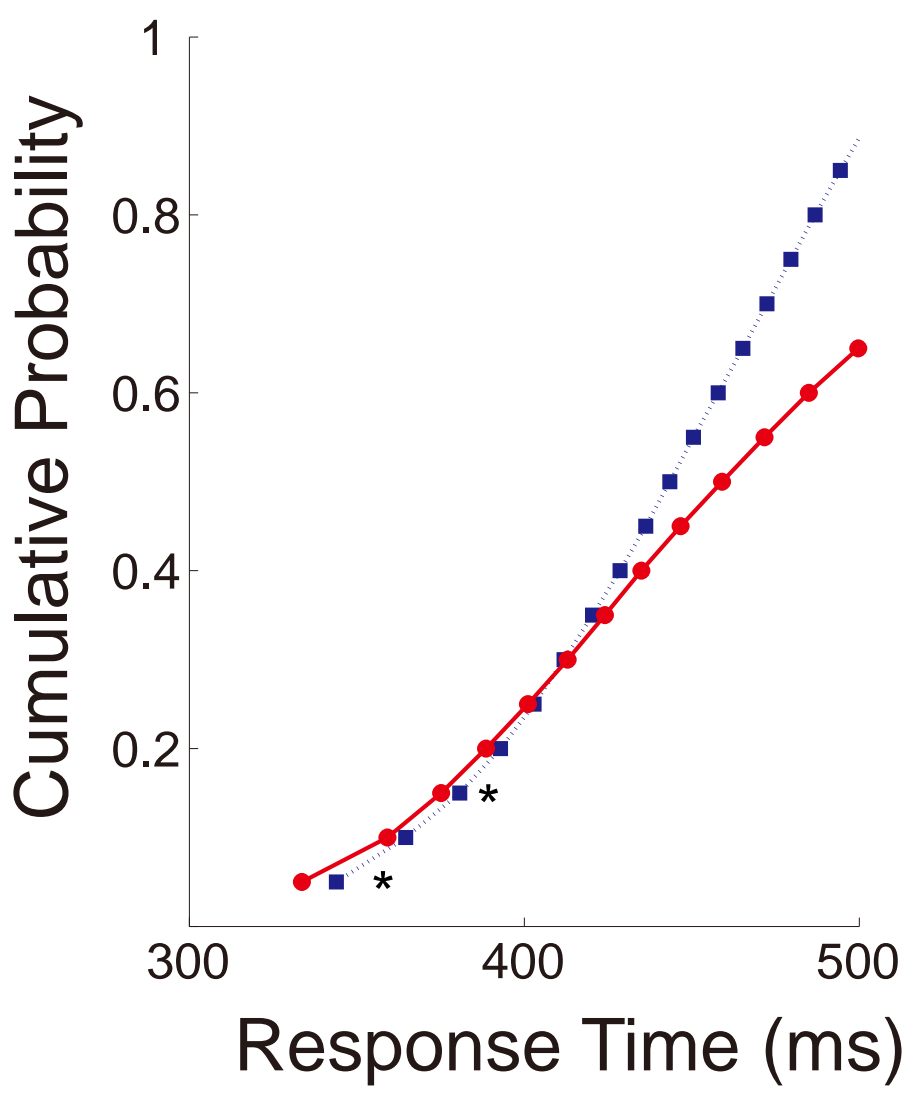

B.

Location-Shared

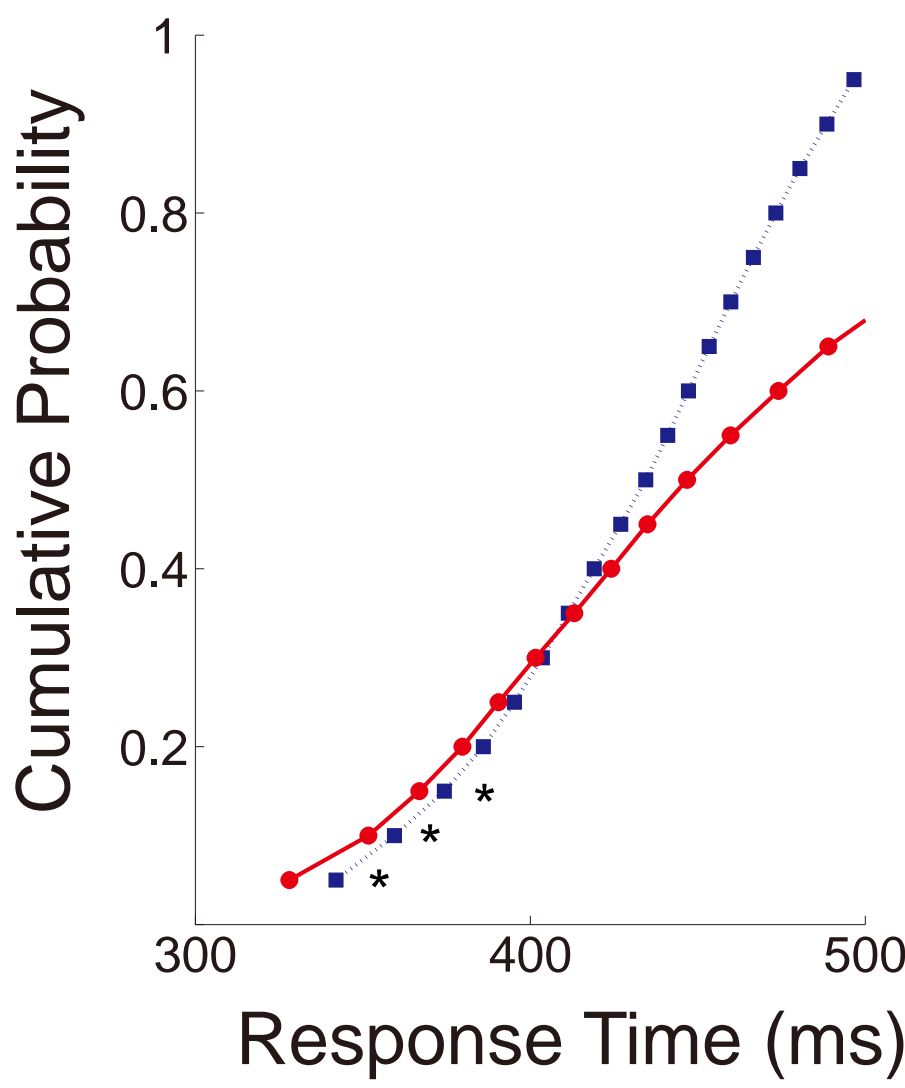

Separated condition

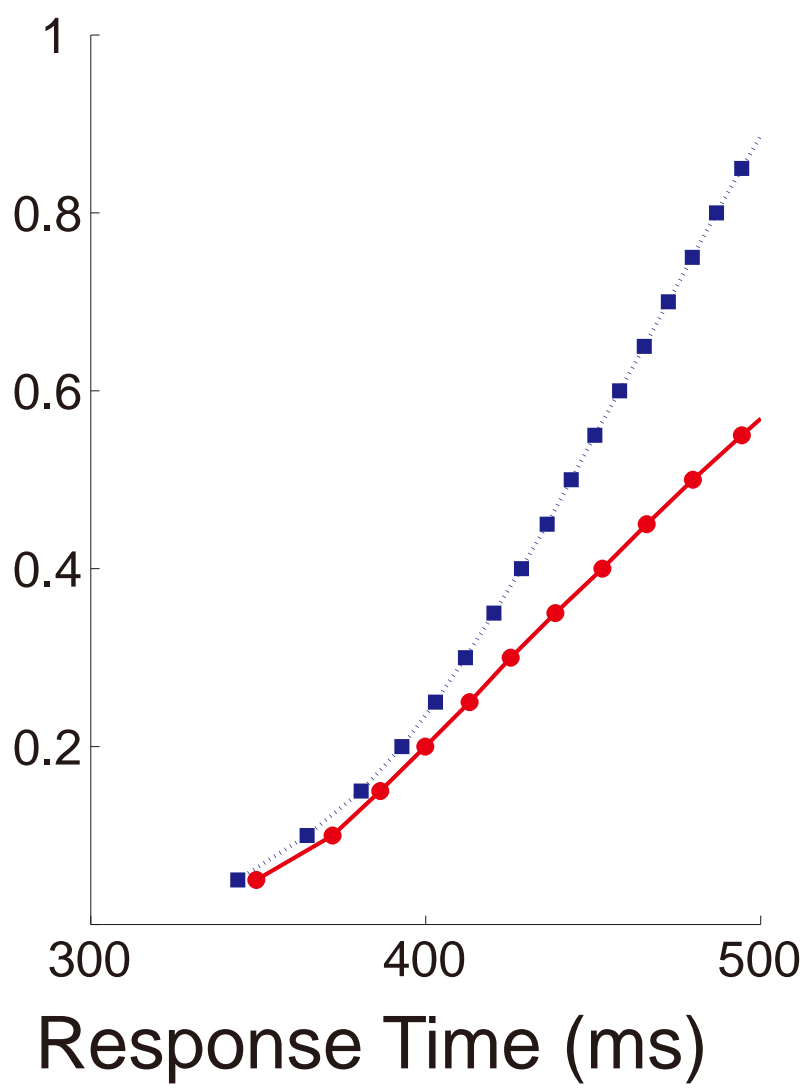

Location-Unshared

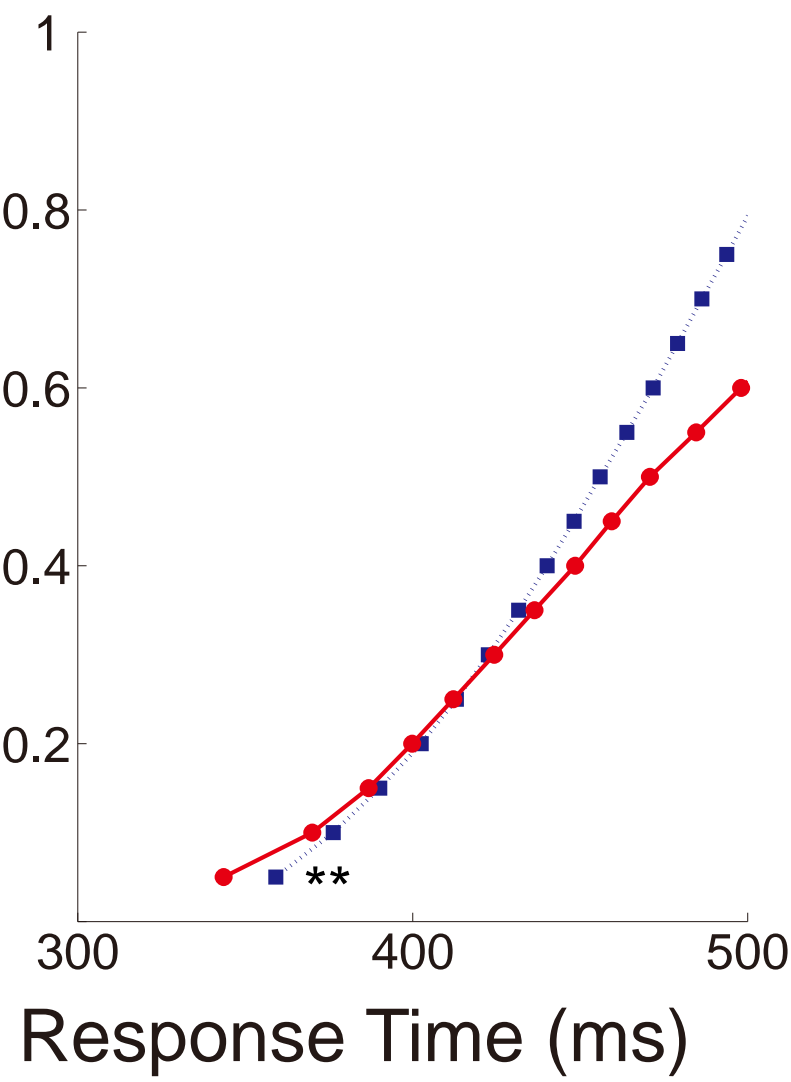

